\title{
Polymeric Lipid Nanosphere Consisting of Water-Soluble Poly(2-methacryloyloxyethyl phosphorylcholine-co-n-butyl methacrylate)
}

\author{
Kazuhiko Ishihara, ${ }^{\dagger}$ Yasuhiko IwaSaKI, ${ }^{*}$ and Nobuo NaKabaYaSHI ${ }^{*}$ \\ Department of Materials Science, Graduate School of Engineering, \\ The University of Tokyo, \\ 7-3-1 Hongo, Bunkyo-ku, Tokyo 113-8656, Japan \\ *Division of Organic Materials, Institute of Biomaterials and Bioengineering, \\ Tokyo Medical and Dental University, \\ 2-3-10 Kanda-surugadai, Chiyoda-ku, Tokyo 101-0062, Japan
}

(Received April 27, 1999)

\begin{abstract}
As water-soluble amphiphilic polymers with the phospholipid polar group (PMB), 2-methacryloyloxyethyl phosphorylcholine(MPC) copolymerized with $n$-butyl methacrylate (BMA), were synthesized. The properties of the MPC polymers in water were investigated by surface tension measurement, ${ }^{1} \mathrm{H}$ NMR and fluorescence spectroscopy, and light scattering measurement. The solubility of the MPC polymers in water depended on MPC unit composition and molecular weight. The surface tension of the aqueous solution depended on the MPC unit composition in the PMB. In the case of poly(MPC), the surface tension did not change as in the case of water at polymer concentrations from $10^{-5} \mathrm{~g} \mathrm{dL}^{-1}$ to $10^{-1} \mathrm{~g} \mathrm{dL}^{-1}$. However, introduction of the BMA unit in the MPC polymer induced decrease in surface tension considerably above $10^{-4} \mathrm{~g} \mathrm{dL}^{-1}$ polymer concentration. This result clearly shows that the PMB forms aggregates in water. According to fluorescence spectroscopy results, the PMB aggregate formed a hydrophobic domain in water and the polarity of the hydrophobic domain was the same as that of $n$-butanol. From the NMR spectra of the PMB in $\mathrm{D}_{2} \mathrm{O}$ at various temperatures, the phosphorylcholine groups were located at the surface of aggregate below $60^{\circ} \mathrm{C}$. The light scattering measurement revealed that the size of the PMB aggregate in water was $23 \mathrm{~nm}$. A hydrophobic fluorescence probe was entrapped in the hydrophobic inside of the PMB aggregate. The PMB aggregate thus quite likely has potential application as a novel drug carrier, to maintain hydrophobic drugs inside polymer aggregates stably.

KEY WORDS Phospholipid Polymer / Polymer Aggregate / Polymeric Lipid Nanosphere / Hydrophobic Domain / Drug Carrier / Blood Compatibility /
\end{abstract}

Molecular assemblies consisting of phospholipids, such as liposomes and lipid microspheres have the potential to carry bioactive molecules in the living organisms because of their excellent blood compatibility. ${ }^{1-3} \mathrm{Al}-$ though many studies have been carried out to realize their effectiveness on drug delivery systems, most efforts failed because of the lack of mechanical and chemical stability. The bioactive molecules entrapped in the inner phase easily leach out shortly after injection. Thus, the stability of these phospholipid assemblies must be improved. Polymerization of these phospholipids seems to be a most effective method for this improvement. ${ }^{4,5}$ However, the polymerization ability of the phospholipids having a diene or acetylene unit is not good and the methacryloyl or acryloyl groups in the phospholipids cannot polymerize using a conventional radical initiator when they form a bilayered structure. As another stabilization method of the molecular assemblies, a combination of polymers with these phospholipid assemblies has been reported. Sunamoto et al. investigated the complexation of a phospholipid liposome with polysaccharide modified with a small amount of hydrophobic cholesterol unit. ${ }^{6}$ The polysaccharide strongly interacted with the liposome and covered its surface. The stability of the modified liposome dramatically increased and could be directly injected in a vein. We tried to modify the liposome with a watersoluble phospholipid polymer, poly[2-methacryloyloxyethyl phosphorylcholine (MPC)], which has the same polar group as the phosphatidylcholines. ${ }^{7}$ The poly-

\footnotetext{
† To whom correspondence should be addressed.
}

(MPC) selectively interacted with dipalmitoylphosphatidylcholine (DPPC) liposome and became attached to the surface. ${ }^{8}$ Thus, the liposomal structure became strong and release of the fluorescent probe was suppressed from the liposome in the presence of the poly(MPC) even in plasma. Since blood compatibility of the liposome treated with poly(MPC) was the same as a normal liposome, it could be used as a carrier of hydrophilic drugs.

MPC can copolymerize with a hydrophobic monomer and water-insoluble copolymers are obtained. ${ }^{9}{ }^{910}$ Protein adsorption and platelet adhesion are suppressed even when blood contacted the surface of the MPC copolymer, that is, the MPC copolymer shows excellent blood compatibility. ${ }^{11-14}$ The blood compatibility is an important property for drug carriers directly injected into the blood stream. From these points of view, we improved the solubility in water of the MPC copolymer for use as a drug carrier for hydrophobic drugs like the lipid nanosphere.

The preparation of the water-soluble MPC copolymer with a hydrophobic moiety and evaluation of its basic properties as a hydrophobic drug carrier are reported.

\section{EXPERIMENTAL}

\section{Materials}

MPC was synthesized as previously reported and recrystallized from acetonitrile. ${ }^{9} n$-Butyl methacrylate (BMA) was of commercial reagent grade from Nacalai Tesque Co., Ltd., Tokyo, Japan. and purified by distillation under reduced pressure and the fraction of 
bp $63^{\circ} \mathrm{C} / 24 \mathrm{mmHg}$ was used. As polymerization initiator, Perbutyl-ND was obtained from Nippon Oil and Fats Co., Ltd., Tokyo, Japan, and used without further purification. Fluorescence probes such as sodium 1-anilino-8-naphthalene sulfonate (ANS), perylene (Pe), pyrene $(\mathrm{Py})$, anilinonaphthalene $(\mathrm{AN})$, and 1,6-diphenyl1,3,5-hexatriene (DPH), were commercially available reagents and used without further purification. All other solvents were distilled by a conventional method.

Preparation and Characterization of Poly $(M P C-c o-B M A)$

Poly(MPC) and poly(MPC-co-BMA)(PMBa: "a" represents the unit mol\% of MPC unit in PMB) with high MPC unit composition were synthesized by conventional radical polymerization in ethanol using $2,2^{\prime}$ azobisisobutyronitrile as an initiator. ${ }^{10}$

PMB with lower molecular weight was synthesized using Perbutyl-ND as an initiator. After polymerization, the reaction mixture was poured into hexane to precipitate the polymer. The polymer was filtered off and dissolved again in ethanol and precipitated in diethylether.

The chemical structures of the obtained poly(MPC) and PMB, were confirmed by ${ }^{1} \mathrm{H}$ NMR and FT-IR spectroscopy. Molecular weight was determined by gel-permeation chromatography (GPC) using the Tosoh system (eluent: chloroform/ethanol=8/2). The numberaveraged molecular weight $\left(M_{n}\right)$ was calculated by comparison of elution times of the sample with poly(ethylene oxide) (PEO) standard. The chemical structure and synthetic results of MPC polymers are indicated in Figure 1 and Table I, respectively.

\section{General Measurements}

The surface tension of aqueous solution containing the MPC polymer at various concentrations was measured by the Wilhermy method with DCA-100 from Orientech, Tokyo, Japan. The temperature was controlled at $22^{\circ} \mathrm{C}$. An ${ }^{1} \mathrm{H}$ NMR spectrum was obtained with a JEOL $\alpha-500$ high-resonance spectrometer. The fluorescence spectrum

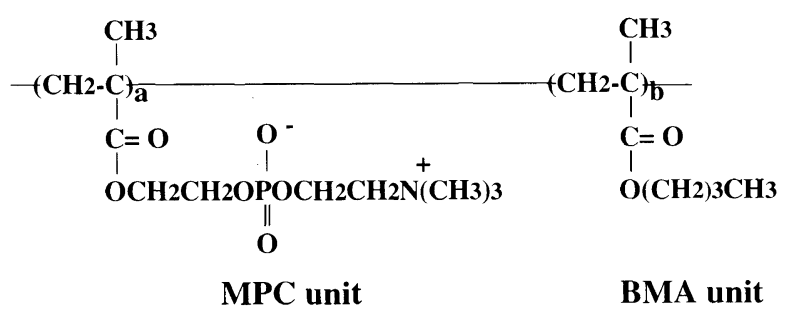

Figure 1. Chemical structure of water-soluble MPC polymer, PMB. was recorded with a FP-750 spectroscope, JASCO, Tokyo, Japan. Light scattering measurement was carried out with a DLS-7000 dynamic light scattering meter, Otsuka Electronics, Osaka, Japan.

\section{Solubilization of Hydrophobic Fluorescence Probe}

The poly(MPC) and PMB30W was dissolved in pure water to make a $0.1 \mathrm{~g} \mathrm{dL}^{-1}$ solution. Excess fluorescence probe was suspended solution and the suspension was shaken $5 \mathrm{~min}$ and then allowed to stand for $15 \mathrm{~h}$ at room temperature (about $21^{\circ} \mathrm{C}$ ). The supernatant was carefully filtered off using millipore filter with $0.45 \mu \mathrm{m}$ pore in diameter and fluorescence intensity of the filtrate was measured at room temperature. The same procedure was carried out using just pure water instead of the polymer solution. The fluorescence intensities of these solutions were compared to determine the solubilization ability of PMB30W.

\section{RESULTS AND DISCUSSION}

\section{Solubility of MPC Polymers in Water}

Since the MPC unit was extremely hydrophilic, a poly(MPC) and PMB with high MPC unit composition could be dissolved in water. The polymer having about 30 unit mol\% of MPC, PMB30, did not dissolve in water, but PMB30W whose molecular weight was low compared with that of PMB30 became water-soluble. This means the solubility of the PMB depends on MPC unit composition and molecular weight of the polymer. The solubility of the MPC unit is quite unique compared with other non-ionic hydrophilic vinyl monomer units such as acrylamide and $N$-vinyl pyrrolidone(VPy). A large amount of hydrophobic BMA units could be solubilized in water by incorporation of only $30 \mathrm{~mol} \%$ MPC units.

\section{Morphology of PMB in Water}

The surface tension of water is very sensitive to solutes. ${ }^{15}$ For example, dissolution of some inorganic ions increases the surface tension of water whereas general organic compounds induce its lowering, depending on the molecular structure or aggregation state of the solute. Measurement of the surface tension of polymer solution is thus useful to evaluate the conformation of polymer in water.

The polymer concentration dependence of the surface tension was investigated in aqueous solution of watersoluble MPC polymers and the results are shown in Figure 2. In the case of poly(MPC), the surface tension

Table I. Synthetic result of MPC polymers

\begin{tabular}{|c|c|c|c|c|c|}
\hline \multirow{2}{*}{ Code } & \multicolumn{2}{|c|}{$\mathrm{mol}^{\%}$ of $\mathrm{MPC}^{\mathrm{a}}$} & \multirow{2}{*}{$\begin{array}{c}\text { Yield } \\
\%\end{array}$} & \multirow{2}{*}{$M_{n} \times 10^{-5 \mathrm{~b}}$} & \multirow{2}{*}{ Solubility in water } \\
\hline & In feed & In polymer & & & \\
\hline Poly(MPC) & 100 & 100 & 89.2 & 0.80 & ++ \\
\hline РМB80 & 80.0 & 71.0 & 69.0 & 3.5 & ++ \\
\hline PMB60 & 60.0 & 52.0 & 61.0 & 2.7 & ++ \\
\hline PMB30 & 30.0 & 27.0 & 79.0 & 3.8 & - \\
\hline PMB30W & 30.0 & 30.0 & 75.0 & 0.54 & ++ \\
\hline
\end{tabular}

${ }^{\mathrm{a}}$ Unit mol\% of MPC for the MPC polymers. ${ }^{\mathrm{b}}$ Estimated by GPC with PEO standard, $M_{n}$ represents the number average molecular weight. ${ }^{\mathrm{c}}$ Solubility was determined by $1 \mathrm{mg} \mathrm{mL}^{-1}$ each polymer sample and described as soluble $(++)$ and insoluble $(-)$. 


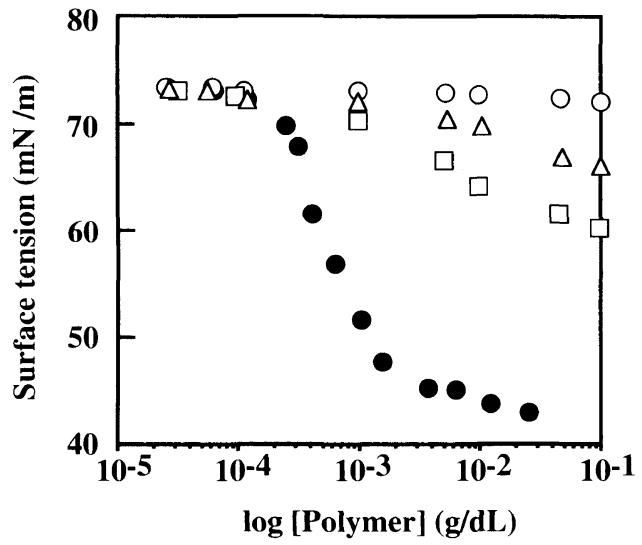

Figure 2. Relationship between concentrations of MPC polymers and surface tension of aqueous solutions at $22^{\circ} \mathrm{C}$. (O) Poly(MPC), $(\triangle)$ PMB80, ( $\square$ ) PMB60, (•) PMB30W.

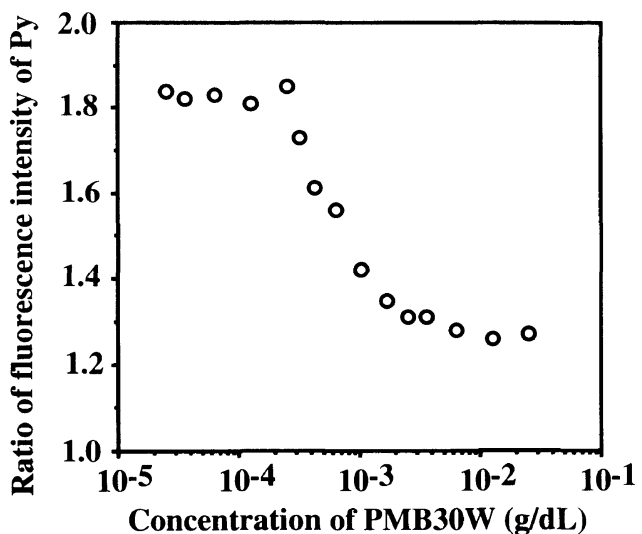

Figure 3. Relationship between concentration of PMB30W and fluorescence intensity of Py solubilized in PMB30W aqueous solution. $[\mathrm{Py}]=4.0 \times 10^{-5} \mathrm{~mol} \mathrm{~L}^{-1}$.

of the solution maintained $72 \mathrm{mN} \mathrm{m}^{-1}$, the value of pure water till the polymer concentration became upto $10^{-1} \mathrm{~g} \mathrm{dL}^{-1}$. Thus, it is considered that the hydrophobic part of the poly(MPC) does not face aqueous medium, because bulky but hydrophilic phosphorylcholine groups located outer side of the poly(MPC) molecule and backbone of the molecules is covered with the phosphorylcholine groups. Every PMB used in this study was surface active. The surface tension began to decrease gradually at $10^{-3} \mathrm{~g} \mathrm{dL}^{-1}$ and more clear with increase in the hydrophobic BMA unit composition in the PMB by comparing the curve of PMB60 with that of PMB80. The surface tension of the PMB30W aqueous solution strongly depended on the polymer concentration. It began to decrease from the $10^{-3} \mathrm{~g} \mathrm{dL}^{-1}$ level and became constant over $10^{-2} \mathrm{gdL}^{-1}$. This indicates that the PMB30W in water aggregates when the concentration is above $10^{-2} \mathrm{~g} \mathrm{dL}^{-1}$.

Figure 3 indicates the ratio of the fluorescence emission intensity of Py at $386 \mathrm{~nm}$ and $480 \mathrm{~nm}$ (concentration of Py, $4.0 \times 10^{-5} \mathrm{~mol} \mathrm{~L}^{-1}$ and excitation wavelength, $342 \mathrm{~nm}$ ). This ratio represents the hydrophobicity of the molecular assemblies in aqueous solution. ${ }^{16}$ The ratio began to decrease with increase in the polymer concentration to $3 \times 10^{-3} \mathrm{~g} \mathrm{dL}^{-1}$ and then became constant at $2 \times 10^{-2} \mathrm{~g} \mathrm{dL}^{-1}$. This polymer concentration dependence was almost the same as that of the surface

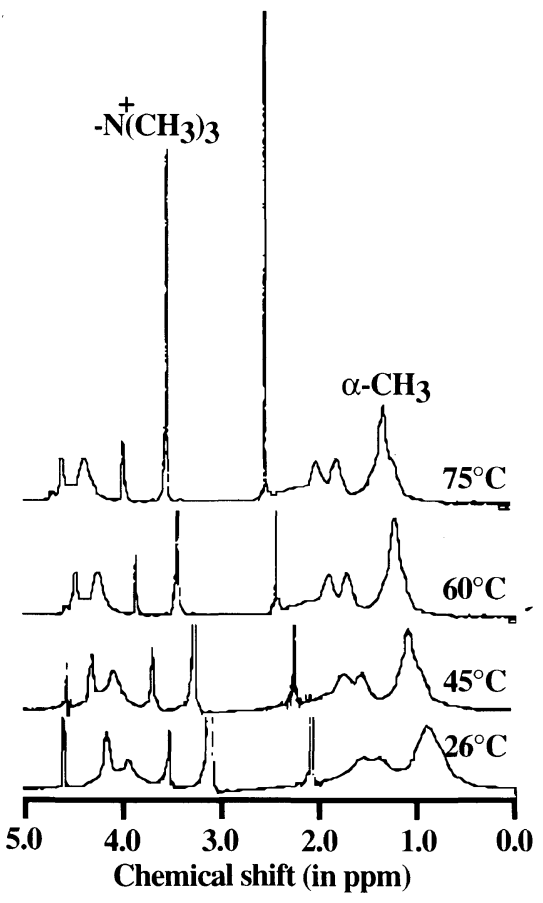

Figure 4. ${ }^{1} \mathrm{H}$ NMR spectra of $\mathrm{PMB} 30 \mathrm{~W}$ in $\mathrm{D}_{2} \mathrm{O}$ at various temperatures. $[\mathrm{PMB} 30 \mathrm{~W}]=0.01 \mathrm{~g} \mathrm{dL}^{-1}$.

tension of PMB30W aqueous solution.

To determine the interaction between the polymer chains of PMB30W in water, ${ }^{1} \mathrm{H}$ NMR spectra were measured at various temperatures. In Figure 4, the representative spectra of $\mathrm{PMB} 30 \mathrm{~W}$ in $\mathrm{D}_{2} \mathrm{O}$ are shown. The characteristic peaks of PMB30W, that is, choline methyl in the side chain and $\alpha$-methyl in the backbone, were observed at $3.0-3.5 \mathrm{ppm}$ and $0.5-1.5 \mathrm{ppm}$, respectively. When the measuring temperature increased, every NMR signal attributed to the PMB30W shifted towards lower magnetic field. This phenomena may be explained by increase in mobility of polymer chain by decrease in viscosity of the polymer solution. However, the NMR intensity of methyl groups in the choline moiety at $3.0-3.5 \mathrm{ppm}$ did not change. The temperature dependence of the NMR intensity attributed to the $\alpha$ methyl of the backbone versus that attributed to choline methyl in the MPC unit is shown in Figure 5. The value was almost constant between 20 to $60^{\circ} \mathrm{C}$, but significantly increased at $75^{\circ} \mathrm{C}$. The intensity of the ${ }^{1} \mathrm{H}$ NMR signal of a functional group was strongly dependent on the environment surrounding the functional group. ${ }^{17}$ If the specific functional group located on the surface of the polymeric aggregate faces the solvent, the intensity of NMR signals of the functional group should be constant. If the functional group is located on the inside of the polymeric aggregate, the intensity of the NMR signals is weak compared with that on the surface. Therefore, the ratio of the intensity of the NMR signal is related to functional groups located on the surface versus those located on the inside of the aggregate and represents the structure of the polymeric aggregate in the water.

As shown in Figure 5, the ratio of NMR signals attributed to the choline methyl versus the $\alpha$-methyl dramatically increased between $60^{\circ} \mathrm{C}$ and $75^{\circ} \mathrm{C}$, corresponding to structural change of the PMB30W aggregates in this temperature range. The hydrophobic 
interaction become weak above $60^{\circ} \mathrm{C} .{ }^{18}$ The structural change of the PMB30W aggregates may correspond to the weakness of the hydrophobic interactions between polymer chains. In other words, the dominant force for aggregation of the PMB30W molecules is hydrophobic interaction. The hydrophobic compounds can then be entrapped and maintained in PMB30W aggregates.

To determine polarity in polymer aggregates composed of PMB30W, fluorescence spectra of ANS in the PMB30W aqueous solution with various concentrations were measured. When ANS exist in a hydrophobic circumstances, enhancement of the fluorescence quantum

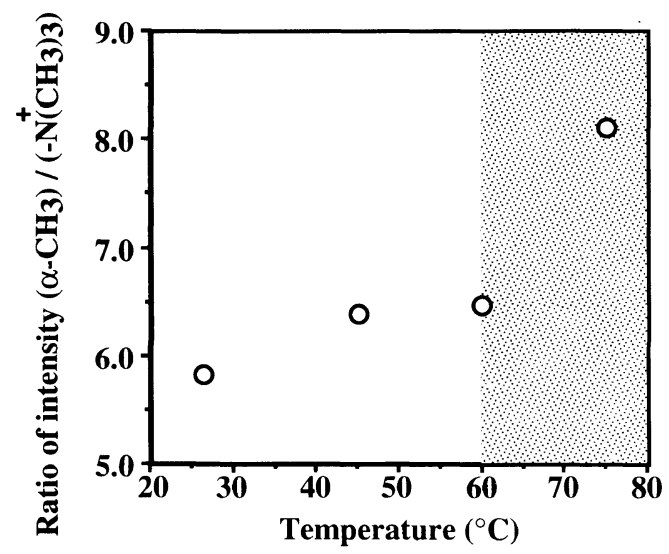

Figure 5. Temperature dependence of ratio of NMR intensity attributed to $\alpha$-methyl group and choline methyl group in PMB30W.

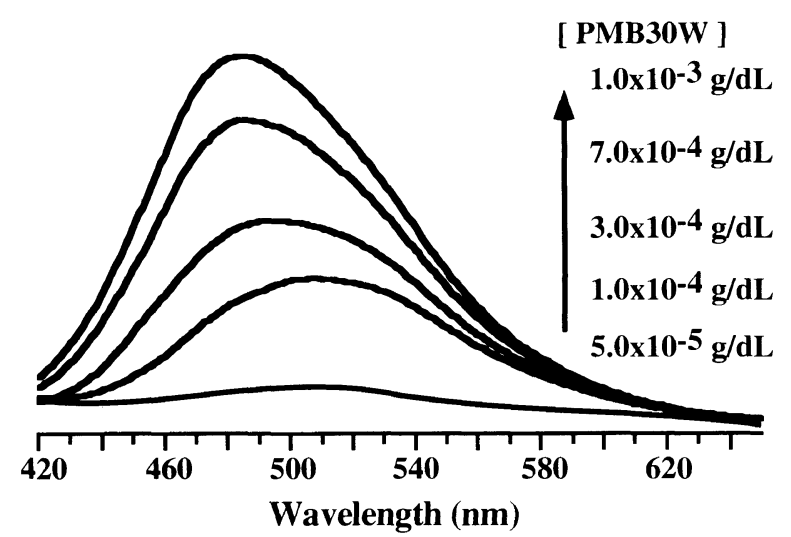

Figure 6. Typical fluorescence spectra of ANS in PMB30W aqueous solution at various PMB30W concentrations. [ANS] $=1.0 \times 10^{-5}$ $\mathrm{mol} \mathrm{L}^{-1}$.

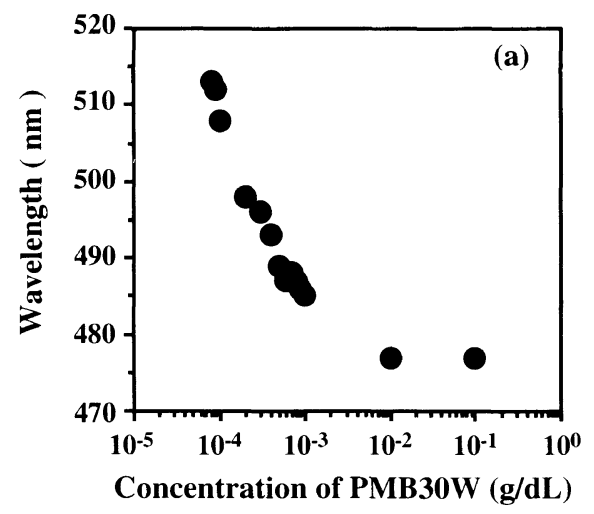

yield and shift of the emission maximum toward the blue are observed. ${ }^{19}$ An ANS molecule can bind only to a hydrophobic region near the aqueous medium like the surface of a surfactant micell or the hydrophobic cleft of globular proteins since a sulfonate group of an ANS molecule has to be in contact with the aqueous medium.

Figure 6 shows typical fluorescence spectra of ANS in aqueous solution of the PMB30W at different polymer concentrations. No fluorescence was observed in ANS aqueous solution without PMB30W or at low polymer concentration below $5 \times 10^{-5} \mathrm{~g} \mathrm{dL}^{-1}$ level. This polymer concentration corresponded to that before the surface tension began to decrease. However, when PMB30W concentration increased, the fluorescence intensity of the ANS solution became strong and peak wavelength became much shorter. The concentration dependence of the peak wavelength of the ANS in PMB30W solution is shown in Figure 7(a), with the same tendency as that of surface tension as shown in Figure 2 and fluorescence behavior of pyrene as shown in Figure 3. This is due to aggregation of the polymer and formation of hydrophobic domains in water by hydrophobic interactions between polymer chains. Figure 7(b) summarizes the relationship between solubility parameter of various solvents and maximum wavelength of ANS. The maximum wavelength decreased with a decrease in the solubility parameter. By comparison of Figure 7(a) with Figure 7(b), good relation between PMB30W concentration and solubility parameter is found, The polarity of inside of the PMB30W aggregate almost corresponds to that of $n$-butanol.

The diameter of the PMB30W aggregate was $23 \pm$ $5 \mathrm{~nm}$, as determined by a dynamic light scattering measurement at $1.0 \times 10^{-1} \mathrm{~g} \mathrm{dL}^{-1}$.

\section{Solubilization of Hydrophobic Compounds with PMB30W Aggregate}

A hydrophobic fluorescent probe could be solubilized in PMB30W aggregates by stirring. Table II summarizes the ratio of fluorescence intensity of the fluorescent probe in an aqueous solution of PMB30W versus that in pure water or in poly(MPC) aqueous solution. Since every fluorescent probe tested here hardly dissolved in water and poly(MPC) aqueous solution, the fluorescence intensity was quite low. On the other hand, a strong

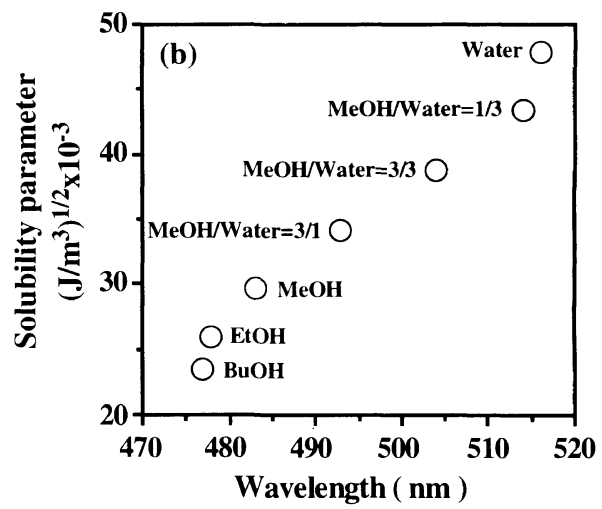

Figure 7. Concentration dependence of the fluorescence peak wavelength of the ANS in PMB30W aqueous solution (a) and relation between solubility parameter of the solvent and fluorescence peak wavelength of the ANS (b). MeOH, EtOH, and BuOH represent methanol, ethanol, and $n$-butanol, respectively. 
Table II. Solubilization of hydrophobic compounds in PMB30W aqueous solution

Compound Ratio of fluorescence intensity

$[$ Polymer $]=0.1 \mathrm{~g} \mathrm{dL}^{-1}$ in water at room temperature.

fluorescence intensity was observed in the presence of PMB30W. Although the fluorescence intensity also increased with decrease in the polarity of the solvent, it is reasonable that the amount of the fluorescent probe dissolved in water increased with the addition of PMB30W. The polar moiety of PMB30W, poly(MPC), could not dissolve the fluorescence probe significantly. The fluorescent probes could locate on the inside of the PMB30W aggregate. The chemical structure of the fluorescent probe did not affect the solubilization of the PMB30W aggregate. This is important because the PMB30W aggregate contains various drugs on the inside of the aggregate.

Liposome and lipid microsphere are unstable in the presence of proteins since they are a molecular assembly of low-molecular-weight phospholipids. Therefore, it is difficult to use them as a drug carrier in the bloodstream for long-term circulation. The PMB30W aggregate, the polymeric phospholipid nanosphere is considered stable under biological conditions and may be used as a drug carrier.

In general, water-soluble polymers with amphiphilic character induce conformational changes of proteins. ${ }^{20}$ Though PMB30W is an amphiphilic polymer, it is considered that a conformation of proteins in PMB30W aqueous solution does not change. We reported that when PMB30, insoluble in water, is coated on a substrate, proteins hardly are adsorbed on the surface, and even when proteins attached on the surface of the substrate, they retain their original secondary structures. ${ }^{21}$ The activity of an enzyme, horse-radish peroxidase (HRP), in the MPC polymer aqueous solution was maintained at the initial level even when stored at room temperature for 20 days. $^{22}$ The reason for such phenomena is not exactly understood at the present time. However, we considered one possibility, that is, the water structure in the MPC polymer solution is quite unique compared with other aqueous solutions containing conventional water-soluble polymers such as poly(VPy) and PEO ${ }^{23}$ In the case of the MPC polymer, bound water on the polymer chains was less than that on the other polymer chains. This means that the MPC polymer interacts with water molecules around the protein molecules quite mildly, and thus does not induce the denaturation of proteins.

Juliano et al. reported interactions between the polymeric phospholipid liposomes and platelets. ${ }^{24,25}$ The coagulation of platelets did not change after the polymeric liposome composed of the phosphatidylcholine derivative was added. The phosphorylcholine group thus has no adverse effect on platelet function. From the NMR spectra of the PMB30W aggregate in $\mathrm{D}_{2} \mathrm{O}$, the surface was found covered with phosphorylcholine groups. PMB30W aggregates with polymeric phospholipid nanosphere morphology may thus not significantly interact with platelets.

\section{CONCLUSIONS}

Amphiphilic polymers composed of MPC units with phosphorylcholine groups and hydrophobic BMA units were prepared. The polymer, PMB30W, was watersoluble when the molecular weight of the polymer was $0.54 \times 10^{5}$, but still a stable aggregate with polymeric phospholipid nanosphere morphology. The aggregate solubilized hydrophobic compounds and stably maintained them. PMB30W should thus prove useful as a drug carrier which can be directly injected into the bloodstream.

Acknowledgments. The authors express appreciation to Mr. Tomohiro Konno, Nihon University for technical support, and Drs. Daijiro Shiino and Ken Suzuki, NOF Co., Ltd. for helpful discussion. This study was partially supported by the Shiseido Science and Technology Research Foundation and a Grant-in-Aid for Scientific Research (B) from the Ministry of Education, Science, Sports and Culture of Japan (No. 09480250).

\section{REFERENCES}

1. H. M. Patel, Biochem. Soc. Trans., 13, 513 (1985). 
2. G. Gregoriadis, Trend Biotech., 3, 235 (1985)

3. Y. Mizushima, Drug Exp. Clin. Res., 11, 595 (1985).

4. S. L. Regen, S. Aingh, G. Oehme, and M. Singh, J. Am. Chem. Soc., 104, 791 (1982).

5. H. H. Hub, B. Hupfer, H. Koch, and H. Ringsdorf, Angew. Chem Int. Ed. Engl., 19, 938 (1982).

6. J. Sunamoto, T. Sato, M. Hirota, K. Fukushima, K. Hiratani, and K. Hara, Biochim. Biophys. Acta, 898, 323 (1987).

7. K. Ishihara and N. Nakabayashi, J. Polym. Sci., Part A: Polym.Chem., 29, 831 (1991).

8. K. Ishihara, R. Tsujino, M. Hamada, N. Toyoda, Y. Iwasaki, and N. Nakabayashi, J. Colloid Interface Sci., submitted.

9. K. Ishihara, T. Ueda, and N. Nakabayashi, Polym. J., 22, 355 (1990).

10. T. Ueda, H. Oshida, K. Kurita, K. Ishihara, and N. Nakabayashi, Polym. J., 24, 1259 (1992).

11. K. Ishihara, R. Aragaki, T. Ueda, A. Watanabe, and N. Nakabayashi, J. Biomed. Mater. Res., 24, 1069 (1990).

12. K. Ishihara, N. P. Ziats, B. P. Tierney, N. Nakabayashi, and J. M. Anderson, J. Biomed. Mater. Res., 25, 1397 (1991).

13. K. Ishihara, H. Oshida, T. Ueda, Y. Endo, A. Watanabe, and N. Nakabayashi, J. Biomed. Mater. Res., 26, 1543(1992).

14. Y. Iwasaki, A. Mikami, K. Kurita, N. Yui, K. Ishihara, and N. Nakabayashi, J. Biomed. Mater. Res., 36, 508 (1997).
15. D. J. Shaw, "Introduction to Colloid and Surface Chemistry," 3rd ed, Butterworths, London, 1980.

16. W. Binana-Limbele and U. P. Strauss, Macromolecules, 23, 2731 (1990).

17. H. Ohno, N. Seki, and E. Tsuchida, Makromol. Chem., 185, 329 (1984).

18. C. Tanford, "Hydrophobic Effect" Wiley-Interscience, New York, N.Y., 1980.

19. S. Ghosh, M. K. Basu, and J. S. Schweppe, Biochim. Biophys. Acta, 337, 395 (1974)

20. S. W. Shalaby, C. L. McCormick, and G. B. Butler, Ed., "Water-Soluble Polymers," American Chemical Society, Washington, D.C., 1991.

21. K. Ishihara, H. Nomura, T. Mihara, K. Kurita, Y. Iwasaki, and N. Nakabayashi, J. Biomed. Mater. Res., 39, 323 (1998).

22. S. Sakaki, K. Ishihara, and N. Nakabayashi, in "Advances in Polymeric Biomaterials Science," T. Akaike, T. Okano, A. Akashi, M. Terano, and N. Yui , Ed., CMC, Tokyo, 1997, p 167.

23. K. Fukumoto, Y. Iwasaki, N. Nakabayashi, Y. Shindo, and K. Ishihara, Polym. Prepr., Jpn., 47, 3048 (1998).

24. R. L. Juliano, M. J. Hsu, D. Peterson, S. L. Regen, and A. Singh, Exp. Cell. Res., 146, 422 (1983).

25. F. Bonte, M. J. Hsu, A. Papp, K. Wu, S. L. Regen, and R. L. Juliano, Biochim. Biophys. Acta, 900, 1 (1987). 